\title{
BIOINFORMATION
}

Discovery at the interface of physical and biological sciences

\section{Correlation of frankfort horizontal with the sella- nasion line angular data in different sagittal skeletal bases}

\author{
Mathew Thomas Maliael, Aravind Kumar Subramanian* \& Remmiya Mary Varghese \\ Department of Orthodontics and Dentofacial Orthopaedics,Saveetha Dental College and Hospital Saveetha Institute of Medical and \\ Technical Sciences (SIMATS), Chennai-600077, Tamil Nadu, India; Aravind Kumar Subramanian - Email ID : aravindkumar@saveetha.com; \\ Corresponding Author*
}

Received October 7, 2020; Revised October 27, 2020; Accepted October 27, 2020; Published December 31, 2020

DOI: $10.6026 / 973206300161088$

The authors are responsible for the content of this article. The Editorial and the publisher has taken reasonable steps to check the content of the article in accordance to publishing ethics with adequate peer reviews deposited at PUBLONS.

Declaration on official E-mail:

The corresponding author declares that official e-mail from their institution is not available for all authors

\section{Declaration on Publication Ethics:}

The authors state that they adhere with COPE guidelines on publishing ethics as described elsewhere at https://publicationethics.org/. The authors also undertake that they are not associated with any other third party (governmental or non-governmental agencies) linking with any form of unethical issues connecting to this publication. The authors also declare that they are not withholding any information that is misleading to the publisher in regard to this article.

\section{Abstract:}

This is part of a special issue on Dental Biology

It is of interest to establish the cephalometric correlation of angular data between frankfort horizontal and the sella-nasion line in different sagittal skeletal bases. Beta angle was used to divide the sample based on their sagittal skeletal base relationship. The FH-SN angle was measured for each group. The data were tabulated into IBM SPSS software. Kolmogorov-Smirnov and Shapiro-Wilk test was done to test the normal distribution of the data. One-way ANOVA analysis was done to test the difference of the FH-SN angle among the groups. Independent samples t-Test was done to test for gender dimorphism. The mean FH-SN angle of the sample was $6.33^{\circ} 3.35^{\circ}$. The results of the One-Way ANOVA and independent samples t-Test were insignificant. Results show that is no statistically significant difference in FHSN angle between skeletal class I, II and III.The mean FH-SN angle of the sample was $6.33^{\circ} 3.35^{\circ}$. The distribution of the data was normal. The results of the One-Way ANOVA and Independent samples t-Test were insignificant. There was no statistically significant difference in FH-SN angle between skeletal class I, II and III.

Keywords: Beta angle; Cephalometry; Frankfort Horizontal; Sella-Nasion line 

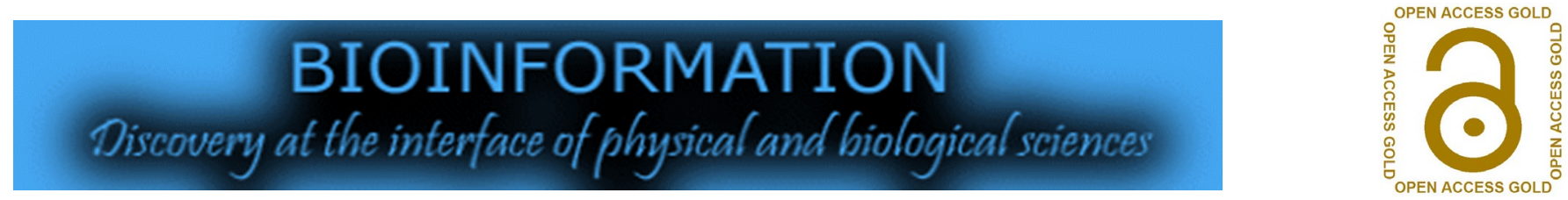

\section{Background:}

Cephalometrics (scientific measure of the dimensions of the head has an important role in orthodontic diagnosis and treatment planning [1-2]. Correlation between the different skeletal structures is known [2]. These analyses often use either the Sella - Nasion (SN) Plane or the Frankfort Horizontal (FH) Plane as a plane of reference [3]. The SN plane is constructed by joining the midpoint of the sella turcica (S) with the Nasion (N) [4]. The FH Plane is constructed by joining the inferior most point in the bony orbit $(\mathrm{O})$ with the superior most point on the external auditory meatus called Porion (P) [3]. The SN plane and the FH plane are considered to be relatively stable even if cephalometric planes show some variations [5]. The angle between the two planes is usually $7^{\circ}$ [6]. Variation in the FH-SN angulation affects cephalometric diagnosis [7]. However, FH-SN angle is not always $7^{\circ}$ and is variable [8-9]. The beta angle described by Baik et al. (2004) to improve sagittal skeletal discrepancies diagnosis is helpful $[\mathbf{1 0}, \mathbf{1 1}]$. The variations in sagittal skeletal relationships have an impact on other skeletal structures. This affects the harmony and balance of the craniofacial complex. Hence, an assessment of the FH-SN angle is essential before arriving at a cephalometric diagnosis. Therefore, it is of interest to document the difference between the FH-SN angles in different skeletal relationships.

\section{Materials and Methods: \\ Dataset:}

53 pre-treatment lateral cephalograms of patients of south Indian descent undergoing orthodontic treatment were collected from the records in the Department of Orthodontics of Saveetha Dental College and Hospital (SDCH), Chennai, India. A single operator using the same equipment took the Cephalograms between July 2019 and December 2019. The patients were within the age bracket of 18 - 34 years, with the average age being 23.58 years. The cephalograms were traced using FACAD version 3.11 (Ilexis AB, Sweden). Landmark points were marked by a single investigator (MTM) to eliminate intra-operator bias. The landmarks were verified by the second investigator (AKS). Then using Beta Angle [11] measurements were used to assess sagittal skeletal discrepancies and divide the cephalograms into 3 groups. The Beta Angle value between $27^{\circ}-35^{\circ}$ indicates a normal Class I skeletal pattern, a beta angle value $<27^{\circ}$ indicates a Class II skeletal pattern, and a beta angle value $>35^{\circ}$ indicates a Class III skeletal pattern. The Class I cephalograms were grouped into Group I, the class II cephalograms were grouped into Group II and the class III cephalograms were grouped into Group III. The reference planes were constructed. The angle between the planes was measured and recorded (Figure 1 - check with authors). Of the 53 cephalograms, 20 were from Group I , 16 were from Group II and 17 were from Group III.

\section{Ethical clearance:}

Clearance for this retrospective study was obtained from the institutional review board of SDCH - The institutional ethical approval number is SDC/SIHEC/2020/DIASDATA/0619-320.

\section{Statistical analysis:}

The Recorded data was entered into IBM SPSS Statistics software version 23 (IBM Corporation, USA). A Kolmogorov-Smirnov and Shapiro-Wilk test was done to test the normal distribution of the data. The mean and standard deviation of the sample was calculated. An independent sample t-Test was done to test the association between gender and FH-SN angle. A One-Way ANOVA analysis was done to assess the association between the FH-SN Plane angle between the groups.

\section{Results and Discussion:}

The overall mean FH-SN angle of the sample was $6.62^{\circ} 3.58^{\circ}$. The values of the Kolmogorov-Smirnov and Shapiro-Wilks tests were above 0.05 , indicating that data was of normal distribution. The mean FH-SN angle was higher in females, yet the difference was not statistically significant. When the means of the FH-SN angle for the three groups were compared using ANOVA analysis there was no statistically significant difference in the FH-SN angle between the groups (Table 1). This study identified the overall average FH$\mathrm{SN}$ angle for the sample to be $6.62^{\circ} 3.58^{\circ}$. The average FH-SN angle among subjects in Group I was $6.65^{\circ} 3.16^{\circ}$, among group II subjects the average was $6.98^{\circ} 3.42^{\circ}$ and the average among Group III was $6.27^{\circ} 4.31^{\circ}$. Among males, the average FH-SN angle was $5.67^{\circ} 3.34^{\circ}$ and among females, it was $7.98^{\circ} 3.53^{\circ}$. The value of the overall average FH-SN angle was close to the accepted norm of the $7^{\circ}$ angle between the FH-SN planes. Although some literature evidence suggests the average value is greater than the norm of $7^{\circ}$ $[6,8,12]$. The significant racial differences in those samples could account for such a variation. The primary objective of this investigation was to evaluate the FH-SN angle in different sagittal skeletal relationships namely: Class I, II, and III. One-way ANOVA analysis revealed that there was no statistically significant difference between the FH-SN angles in different skeletal relationships (Table 1). This is concurrent with findings of Giri et al. [9-13] wherein no statistically significant difference was appreciable between the different skeletal relationship groups. 

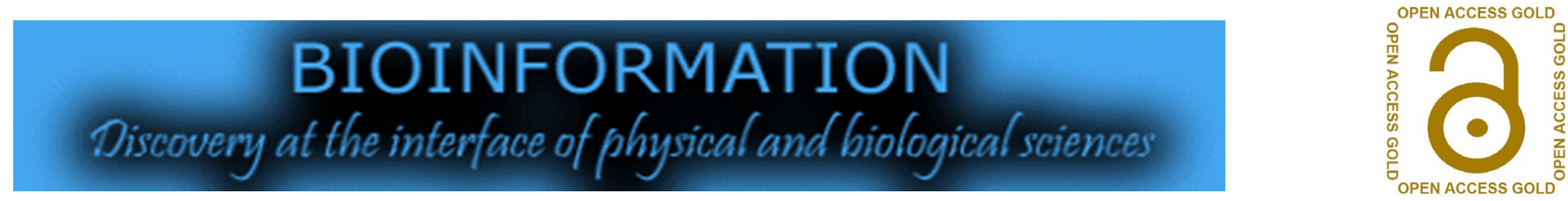

The finding in our investigation is also concurrent with the result obtained by Alves et al where the FH-SN angle was observed to be greater in skeletal class II when compared to skeletal class III $[\mathbf{1 0 , 1 3}]$. Giri et al and Alves et al had used ANB angle and Wits appraisal respectively to assess sagittal skeletal discrepancy. In the case of Wits appraisal introduced by Jacobson, the functional occlusal plane at times is difficult to identify and is not always reproducible in some cases. Also in some situations, the normal development of the dentition or orthodontic intervention can change the angulation of the functional occlusal plane [14]. ANB angle is another popular choice for assessment of sagittal skeletal discrepancies. Various authors have demonstrated that there is some variability between the interpretation of this angle and the actual discrepancy between the jaw bases $[15,16]$. Evidence in the literature shows that position of the nasion is not always fixed during growth $[\mathbf{1 5}, \mathbf{1 7 ]}$ and any displacement in the position of the nasion will affect the ANB angle [18]. Furthermore, jaw rotation due to growth or orthodontic intervention could result in a change in the ANB angle value [16]. Also, the presence of Nasion in both ANB angle and SN-FH angle could result in a confounding bias $[8,12]$. So in our study, we decided to utilize the Beta angle described by Baik et al. [11] to evaluate the sagittal skeletal relationship among the patients because beta angle measurements are independent of errors in identifying landmarks or planes as it is independent of cranial landmarks and the dental occlusion [10]. Furthermore, it is stable even when the jaws are rotated which could camouflage the sagittal discrepancies. Variability of the FH$\mathrm{SN}$ angle among different individuals is quite evident, although whether the value of the angle varied within an individual is debatable. The angle is generally observed to be constant throughout an individual's life, although studies have reported an increase in the angle with age $[8, \mathbf{1 2}, \mathbf{1 6}]$. The present study design, which is limited to a retrospective cross-sectional nature, is unable to address this query. Thus there is a need for more longitudinal studies with adequate samples to assess age-related variations in the FH-SN angle. In our study we were able to observe that the female group had a higher mean FH-SN angle value than compared to males (Table 2 and 3: Figure 2 - check with auhors), Huh et al and Giri et al had reported similar findings in their studies [9,12]. However, this observation was not significant statistically. A cephalometric difference of less than $2^{\circ}$ is generally considered clinically insignificant. Cephalometric diagnosis of an orthodontic case is greatly affected by variations in the FH-SN angle. A study conducted by Moore shows that as the value of the angle between $\mathrm{SN}$ and $\mathrm{FH}$ planes increases there is a decrease in the value of the SNA and SNB angles [19]. Changes in the inclination of the SN plane, FH plane, or both lead to a variation of the FH-SN angle. It is thus imperative that FH-SN angle value must be evaluated before concluding the cephalometric diagnosis. Cephalometric reference planes tend to be variable and poorly related [9]. Therefore, the cephalometric analysis must be conducted using two or more reference planes. It should be noted that perpendiculars to SN and FH planes are alternatively used in diagnosis [20].

\section{Conclusion:}

The mean FH-SN angle for the sample was found to be $6.62^{\circ} 3.58^{\circ}$. The difference between the FH-SN angles among the 3 groups was not significant statistically. Although we could not establish any difference in the value of the FH-SN angle among the groups, we were able to observe that there was some variability in the angle formed between the FH and SN planes and that this variability affected the outcome of the cephalometric diagnosis. Therefore, the FH-SN angle must be evaluated for every case before concluding a cephalometric diagnosis. Females had higher mean FH-SN angle as compared to males in the population.

\section{Conflict of interest:}

The authors would like to inform you that there is no conflict of interest in this investigation.

\section{References:}

[1] https://onlinelibrary.wiley.com/doi/abs/10.1002/hed.28900 80512

[2] Zebeib AM and Naini FB American Journal of Orthodontics and Dentofacial Orthopedics. 2014 p740.

[3] https://www.elsevier.com/books/contemporaryorthodontics-6e-south-asia-edition/proffit/978-81-312-5691-6

[4] https://books.google.co.in/books/about/An_Atlas_and_Ma nual_of_Cephalometric_Rad.html?id=W1FqAAAAMAAJ\&re dir_esc $=y$

[5] Jacobson A, American Journal of Orthodontics and Dentofacial Orthopedics. 1988 p527.

[6] Greiner P et al. J Orofac Orthop. 2004 65:217.[PMID:15160248]

[7] Leitao P and Nanda RS American Journal of Orthodontics and Dentofacial Orthopedics. 2000 117:406. [PMID: 10756266]

[8] Alves PVM et al. The Journal of Craniofacial Surgery. 2008 p211.

[9] Giri J et al. Orthodontic Journal of Nepal. 2017 p14.

[10] Jajoo A et al. A Cephalometric Study. J Contemp Dent Pract. 2018 19:739. [PMID: 29959305]

[11] Baik CY and Ververidou M Am J Orthod Dentofacial Orthop. 2004 126:100. [PMID: 15224065] 


\section{BIOINFORMATION \\ Discovery at the interface of physical and biological sciences}

[12] Huh YJ et al. The Angle Orthodontist. 2014 p286.

[13] Alves PVM et al. Journal of Craniofacial Surgery. 2008 19:334. [PMID: 18362708]

[14] Richardson M. Eur J Orthod. 1982 4:251.

[15] Freeman RS Angle Orthod. 1981 51:162. [PMID: 6942667]

[16] Jacobson A, American Journal of Orthodontics. 1975 p125.
[17] Moore AW American Journal of Orthodontics. 1959 p399.

[18] Binder RE J Clin Orthod. 1979 13:258.

[19] Moore JW J Oral Surg. 1976 34:24. [PMID: 1059748]

[20] Incisivo $V$ and Silvestri A Journal Of Craniofacial Surgery. 2000 p31

Edited by $P$ Kangueane Citation: Maliael et al. Bioinformation 16(12): 1088-1092 (2020)

License statement: This is an Open Access article which permits unrestricted use, distribution, and reproduction in any medium, provided the original work is properly credited. This is distributed under the terms of the Creative Commons Attribution License

\section{Articles published in BIOINFORMATION are open for relevant post publication comments and criticisms, which will be published immediately linking to the original article for FREE of cost without open access charges. Comments should be concise, coherent and critical in less than 1000 words.}




\section{BIOINFORMATION}

Discovery at the interface of physical and biological sciences
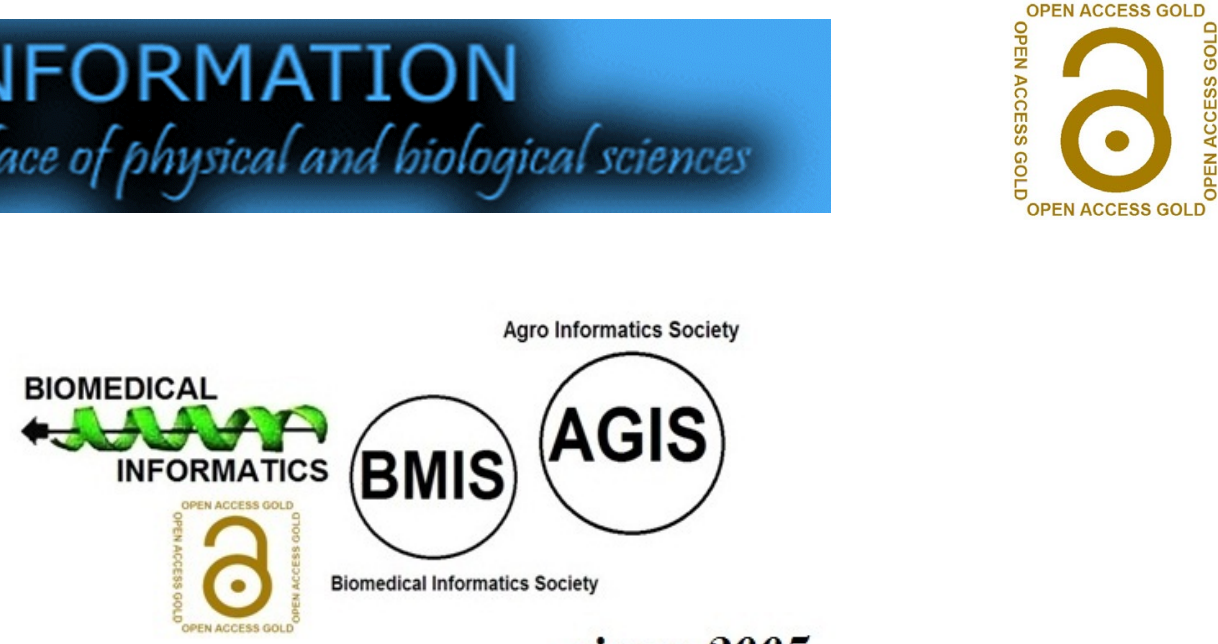

since 2005

\section{BIOINFORMATION}

Discovery at the interf ace of physical and biological sciencess

\section{indexed in}

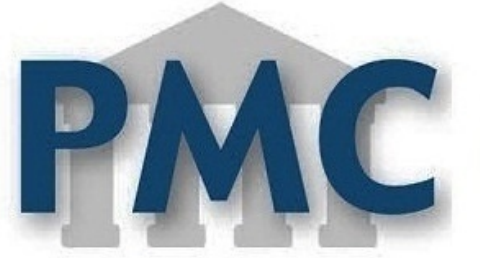

PublMed

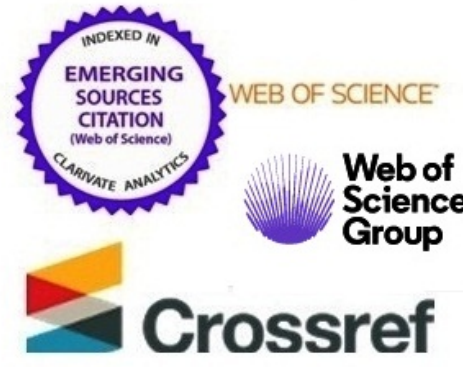

EBSCO

ResearchGate
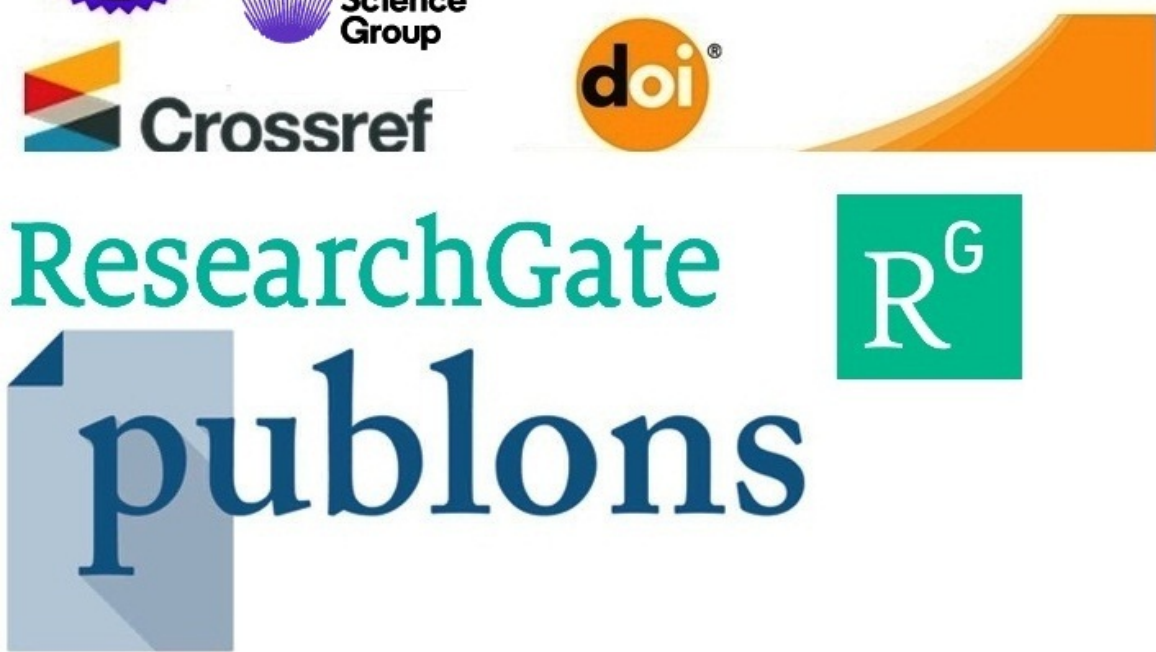